\title{
Cartridge-based Automated Synthesis - A New Tool for the Synthetic Chemist
}

\author{
Benedikt M. Wanner*, Paula L. Nichols, and Tuo Jiang
}

\begin{abstract}
Despite recent advances in reaction methodologies, organic synthesis remains complex and challenging. Many of the fundamental processes in use have not changed in over 100 years, with a large proportion of the work being carried out manually, using lengthy procedures and difficult or hazardous reaction conditions. As such, organic synthesis still presents a bottle-neck in discovery research. Endeavours to automate synthesis in discovery, through robotic platforms, have so far not been widely successful because the highly complex nature of such machines, and the level of skill required for their operation, presents a barrier too great for most discovery chemists. Synple Chem has developed a safe, easy to use, efficiency-enhancing automated technology for the acceleration of discovery research. The automated flow-batch hybrid system utilises a range of innovative pre-packed reagent cartridges for different reaction classes, along with pre-programmed, highly optimised but editable reaction protocols. The combination of these three key elements, provides users with a convenient, easy to use, time-saving technology that makes the synthesis of molecules far simpler, faster and more efficient. The described technology offers all discovery chemists access to real synthesis automation without any of the barriers that have previously restricted its utility.
\end{abstract}

Keywords: Automated synthesis · Discovery chemistry $\cdot$ Laboratory automation $\cdot$ Medicinal chemistry · Synthetic chemistry

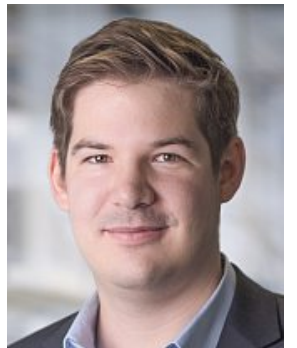

Benedikt M. Wanner graduated from ETH Zurich in the group of Professor Jeffrey W. Bode and has started developing Synple Chem's technology during his time as a $\mathrm{PhD}$ student. For this business idea he was awarded with the ETH pioneer fellowship for the development of the idea into a viable product. Since the company was founded in 2016 he is serving as CEO and co-founder of Synple Chem.

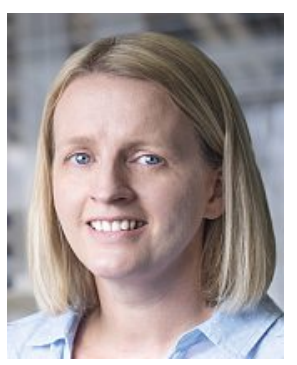

Paula L. Nichols earned her PhD degree in 2003 in the group of Professor Nigel Simpkins at the University of Nottingham and joined GlaxoSmithKline (GSK) as a medicinal chemist. She spent her time at GSK working on numerous projects in the neuroscience, gastrointestinal, and pain therapy areas before moving to Eisai in 2010. Following a move to Switzerland, she joined ETH Zurich and co-founded Synple Chem, where she is currently working in business development.

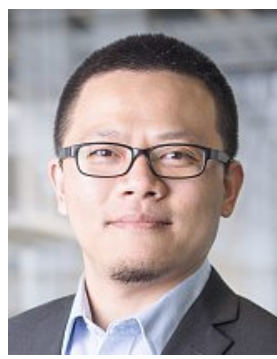

Tuo Jiang obtained his $\mathrm{PhD}$ degree from Professor Jan-E. Bäckvall's group at Stockholm University, Sweden and has more than 10 years experience in synthetic chemistry from four research laboratories in Asia and Europe. He joined and co-founded Synple Chem in 2016 and has since been leading the development of new applications and cartridges.

\section{Addressing the Automation Needs of Discovery Chemistry}

The synthesis of molecules with diverse chemical structures plays a key role in the discovery of new drugs and materials. Despite this, most of the current processes remain expensive, laborious, time- and resource-consuming, environmentally unfriendly, and prone to safety liabilities. As a result, a large portion of synthesis is now outsourced to overseas providers of synthetic services. However, this introduces delays and risks in the drug discovery process, where speed and flexibility are of upmost importance. Without the flexibility to explore and react to emerging biological data in-house, the opportunity to exploit serendipitous discoveries can be lost. ${ }^{[1]}$

Delays can significantly impact drug discovery, where the search for new drugs requires many design, synthesis and screening iterations. A slow feedback loop between preparing and testing molecules can prolong the already lengthy drug discovery process. In an industry burdened with productivity pressures and spiralling costs, there is an enormous demand for new automated technologies that will improve productivity while controlling costs. ${ }^{[2]}$

With an awareness of the need for easy to use automation tools to relieve the synthetic chemistry bottle-neck in discovery research, Synple Chem was founded in 2016 with the aim of introducing safe, easy to use, time-saving technologies for chemical synthesis that enable chemistry-based research and development organizations to simplify chemical synthesis and accelerate the drug discovery process by speeding up the feedback loop between preparing and testing molecules (Fig. 1). Our goals align with the strategy adopted by automated purification system providers, in that we focus on developing technologies with low complexity and a limited number of operations that it performs very well, with a high degree of reliability, e.g. chemical transformations frequently used in discovery chemistry, for which we can provide dedicated pre-packed reagent cartridges. 


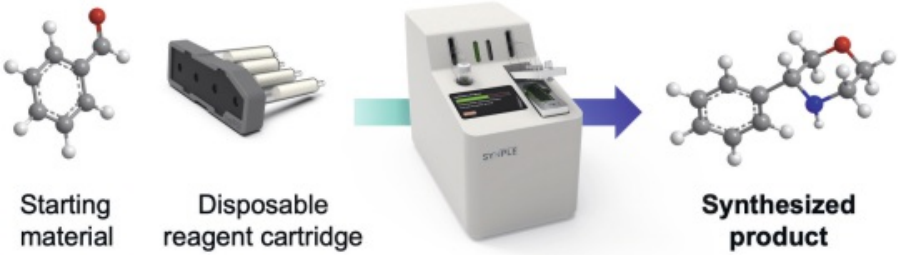

Fig. 1. The Synple Chem systems can transform chemical starting materials into the desired synthetic products using single-use pre-filled reagent cartridges, which are available for a range of chemical reactions.

\section{Pre-optimised, Automated Cartridge-based System}

Despite previous efforts to automate synthesis through robotic platforms, these technologies have so far not been widely adopted in discovery research because the highly complex nature of such machines and the level of skill required for their operation present a barrier too great for most discovery chemists to warrant the investment of their time. Therefore, Synple Chem sought to take an alternative approach by developing a very simple system that would be easy to use and require minimal hands-on time to operate.

As such, a small $(26 \mathrm{~cm} \times 49 \mathrm{~cm})$ automated flow-batch hybrid system has been developed, which utilises a range of innovative pre-packed reagent cartridges for different reactions, along with pre-programmed, highly optimised reaction protocols with editable parameters that can be loaded by simply scanning the cartridge (Fig. 2). The small, bench-top instrument works in an analogous manner to well known household capsule coffee systems and combines the same three key elements that guarantee simplicity and convenience - the automated synthesiser (coffee machine), reagents cartridges with high quality QC checked materials (coffee capsules) and the pre-optimised reaction protocols (automated sequence for the ideal coffee preparation process).
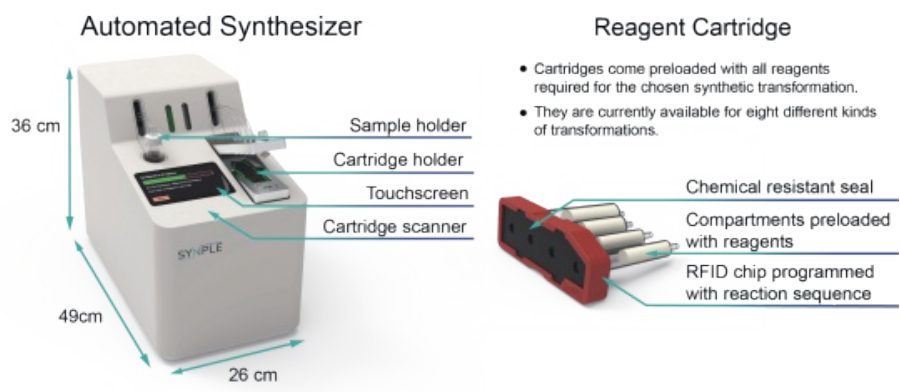

Fig. 2. Setup and features of the automated cartridge-based synthesiser - Synple 2 (on the left) and the reagent cartridge (on the right).

A key difference to the coffee systems, which use only one starting material (water), is that many unique, bespoke molecules can be prepared at the touch of a button since a broad range of starting materials are well tolerated. The user simply loads the starting material into the sample holder and selects an appropriate cartridge for the transformation from the range of available reagent cartridges (Fig. 3).

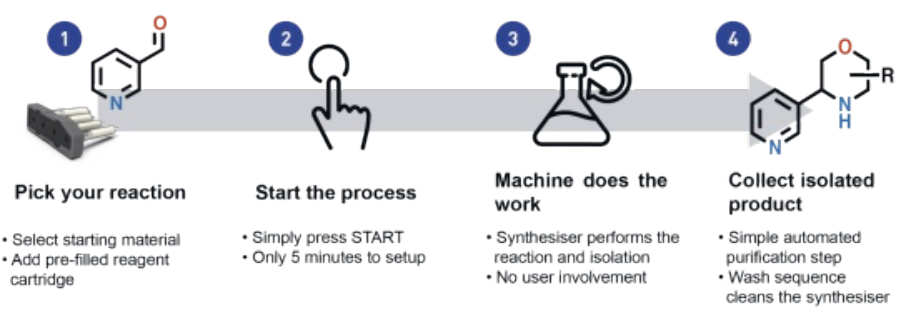

Fig. 3. Workflow of running a reaction on the Synple system in four easy steps.
Each of our cartridges comes pre-filled with all the required reagents for the transformation and purification and also contains an RFID chip that codes for the necessary reaction sequence, thus simplifying reaction set up and minimizing operational error. The majority of the cartridges can be used for any amount of starting material, up to a maximum of $0.5 \mathrm{mmol}$ (except for chemical biology applications, which are $0.1 \mathrm{mmol}$ ), which is a scale compatible with the needs of discovery chemistry.

After scanning the cartridge, the method for that specific reaction is automatically loaded. Although the pre-optimised methods are developed to cover a wide scope of starting materials, the user also has the option and flexibility to easily edit defined reaction parameters, should their starting materials fall outside of the scope of the reaction method. To initiate the chemical reaction, the user simply inserts the cartridge and presses start on the touch screen. From this point, no user involvement is required until the end of the reaction, at which point the sample vial can be removed, the solvent evaporated to give the purified product, and the disposable cartridge properly discarded or recycled. The instrument is also programmed to self-clean, meaning it is ready for use again shortly afterwards, much like modern, cartridge-based automated chromatography systems or coffee makers.

The current synthesiser, Synple 2 - the second generation machine launched by Synple Chem in October 2019, differs primarily from the first generation in that solvents are stored externally to the machines, thus making it a much more compact unit. Another advantage of Synple 2 is that each unit can be used as a module forming part of a flexible parallel synthesiser, which can be tailored in size to meet the customer requirements. With all the modules controlled via a central console, the customer can start off with a relatively small parallel reactor system and add further units to this, as and when needed. Also, if there is a problem with one module, this can be easily isolated and the rest of the system continues working as normal so that there is rarely any downtime due to service, repair and maintenance (Fig. 4).

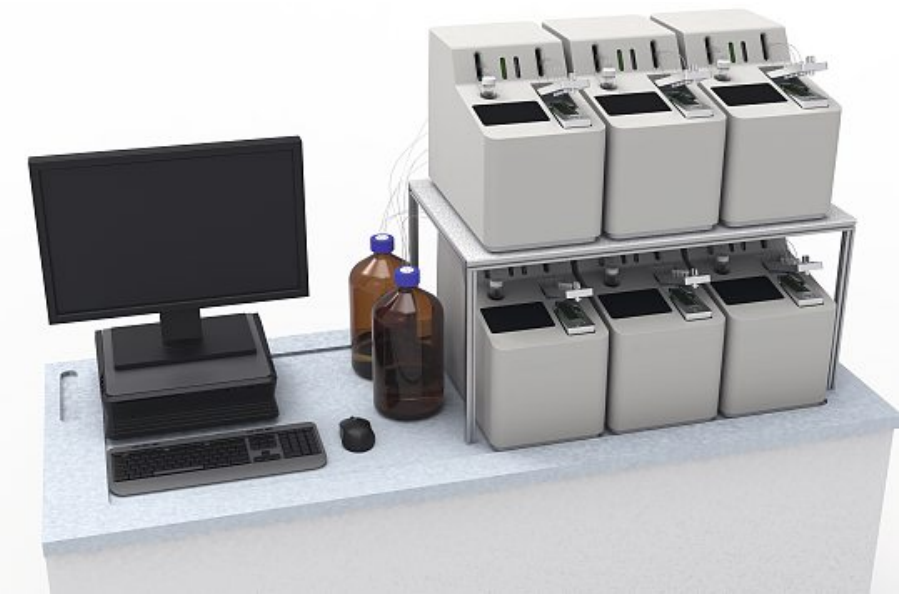

Fig. 4. The Synple Unity - a flexible, module-based parallel synthesis system.

Although the underlying technology in all versions of the machine functions in exactly the same quick and easy to use manner, the Synple 2 and Synple Unity systems have enhanced software features compared to the Synple 1. Instead of being restricted to just the pre-installed methods developed by Synple Chem, the user can also easily adjust defined parameters within each method, e.g. the reaction time and temperature for each step, or exercise the option to skip the purification step, in case there would be any concerns regarding stability of the product. This effectively allows the user to define their own methods more suited to their specific starting materials, with a very minimal amount of effort. 
In order to determine if method modifications might be required, the user can refer to the extensive set of application notes available for each reaction class. Within these notes, the full scope of the reaction in terms of substrates, solvents, temperatures etc. is detailed, as well as trouble shooting hints and tips. A set of instruction cards also accompany the machine, which show in a very simplistic way, how to set up each class of reaction, thus making it incredibly easy to set up and run reactions using this technology (Fig. 5).

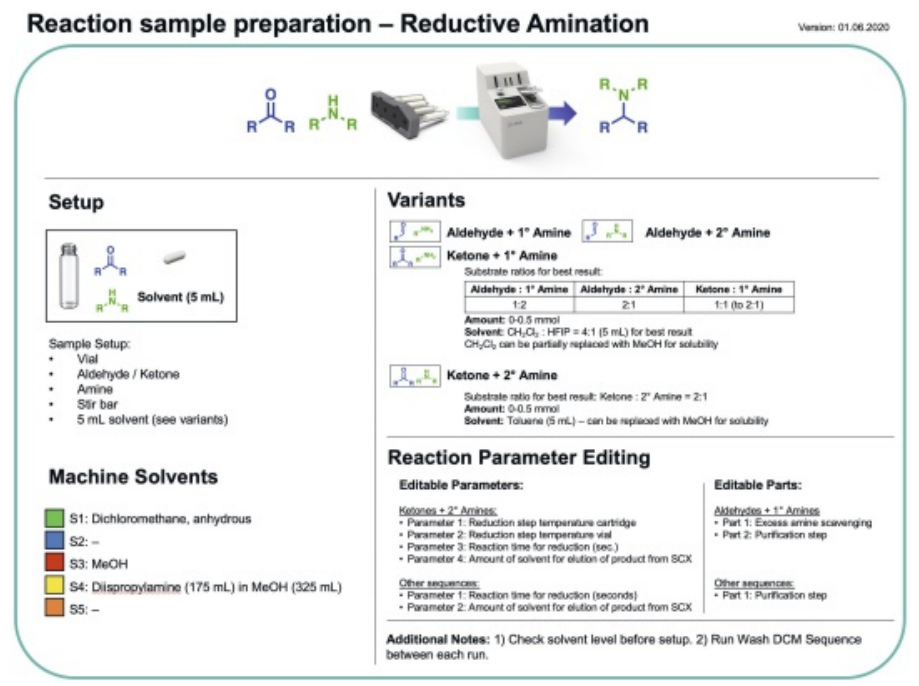

Fig. 5. An example set-up instruction card, which is provided with the machine.

\section{Reaction Applications and Processes}

In order to achieve the greatest utility from the technology, our goal is to develop cartridges and automated protocols for a large proportion of the most commonly used reactions in discovery chemistry. As such, careful reaction sequence development and identification of the most appropriate reagents for the broadest scope of substrates within each reaction class, has been a major focus of our research. The reaction conditions and reaction times are optimised to give a procedure suitable for use with most substrate combinations, and give good yields and purities. When necessary these can also be easily fine-tuned (via the editable reaction parameters) by the user to ensure the maximum yields and purities for their specific substrates.

The current range of single-use reagent cartridges encompasses eight different reaction classes (Fig. 6). These reaction applications include those that enable the preparation of important chemical products, including saturated $N$-heterocycles ${ }^{[3]}$ - currently one of the most desirable motifs for medicinal chemists in drug discovery, plus PROTACs, ${ }^{[4]}$ one of the hottest new trends in drug discovery. Other available cartridges are continually undergoing expansion, based on feedback from discovery chemists and the Synple user community. Targeting the most commonly used reactions in discovery, we have also developed cartridges for Mitsunobu reactions, protecting group chemistry, and reductive aminations.

Reductive aminations, for example, are one of the most widely utilised reactions in discovery chemistry because the resulting amine products, which form via the reaction of a carbonyl group and an amine, are important features in many biologically active molecules. Using the Synple system and cartridges, the user simply selects the two reaction partners, the aldehyde or ketone and the amine, and adds them both to the reaction vial, together with the reaction solvent. The first compartment of the reagent cartridge contains a solid-supported cyanoborohydride and the second holds a proton source (phenylacetic acid). Upon initiation of the reaction, the automated machine sequence pumps the reaction

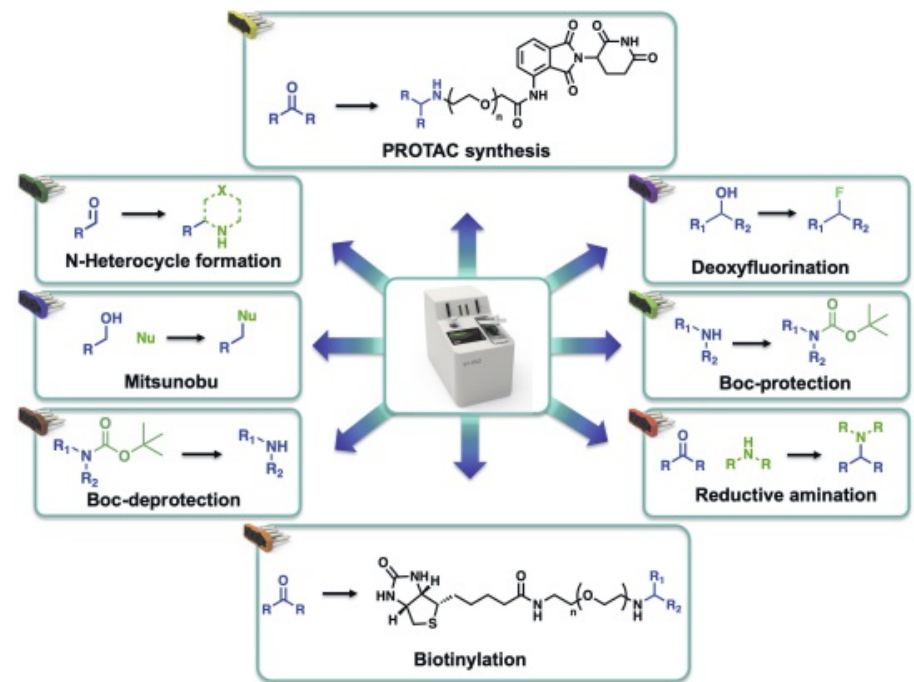

Fig. 6. Range of available reaction classes and cartridges for the Synple system.

solution through the second compartment to dissolve the proton source and then subsequently through the first to effect the reduction of the newly-forming imine. Once these reaction steps are complete, the reaction solution is automatically progressed to the isolation/purification steps and is sequentially passed through the third and fourth compartments, containing a catch \& release resin (SCX) and a scavenger for unreacted starting materials respectively. Using only 5-10 minutes 'hands-on' time, a wide variety of compounds can be synthesized using the pre-installed, optimised conditions, leading in most cases to high yields and purity after 3 to 5 hours reaction time (Scheme 1).

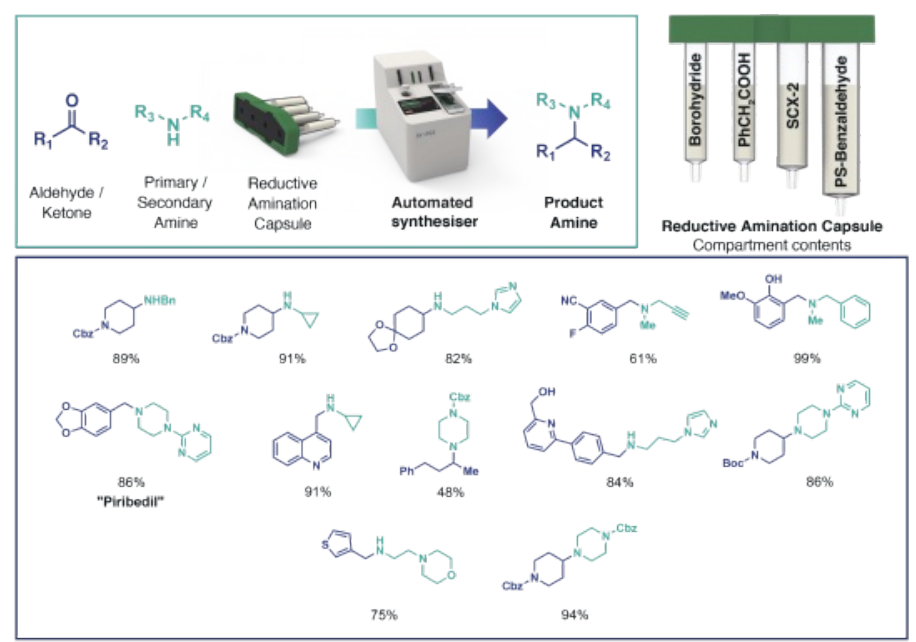

Scheme 1. Reductive amination process and products. Aldehydes and ketones together with primary or secondary amines can be converted in the product amine by using the reductive amination cartridge. Yields are given in percent (\%).

All of our other reaction classes are typically set up in a similar manner, with 1-2 compartments containing the reagents needed to effect the transformations and 1-2 compartments containing the materials needed for product isolation and purification. A full set of detailed application notes for each reaction class are available for download. ${ }^{[5]}$

\section{Benefits of Cartridge-based Automated Synthesis}

Use of the technology offers several key advantages for the discovery chemists, specifically with respect to effective use of time, costs, safety and productivity (Fig. 7). By employing our 
technology, which requires only 5-10 minutes of 'hands-on' time per reaction, the time a researcher spends making a given molecule can be reduced by up to $80 \%$. As such there is a big impact on costs as researchers are free to carry out other tasks while the system automates their reactions. In addition, given the highly optimised processes employed, up to $90 \%$ less waste is produced. This also impacts costs and it is anticipated that a given molecule can be prepared at $30 \%$ of the cost of traditional manual synthesis when using Synple's technology.

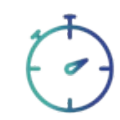

10x More efficient

...compared to manua synthesis. Maximum $10 \mathrm{~min}$ to setup the reaction

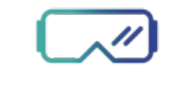

No exposure to toxic chemicals Fully enclosed reaction setup.

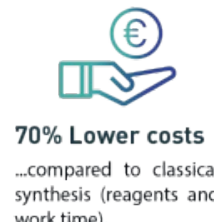
work time).

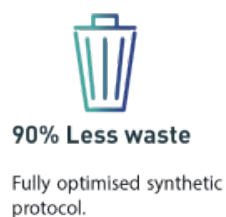

optimised synthetic protocol.

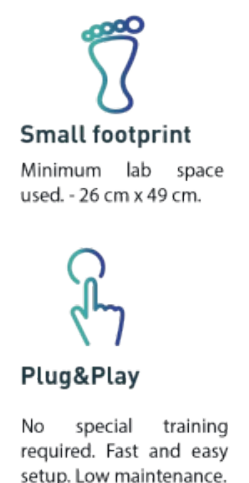

Fig. 7. Advantages of running chemical synthetic reactions with the Synple system compared to traditional manual synthetic chemistry.

Since all the reagents and purification materials are enclosed within the cartridge the quality of the reagents is assured and each cartridge offers a 'fresh' batch of reagents for each reaction, unlike traditional reagent stocks, which can become contaminated and degrade after multiple uses. In addition, the user has no need to handle and weigh out potentially toxic materials. During the course of the reaction, all the reagents are contained within the system and upon completion all the waste remains contained either within the cartridge or in the waste solvent bottle, both of which can be disposed of in the standard way. As such, the user's exposure to toxic agents is greatly minimized, thus the technology provides a safer alternative to traditional synthesis.

While most automation aims to remove the human element to some extent, with chemical synthesis and discovery research the real advantages come from the combination of the human element and machine automation. The Synple system uses automation to do what it does best - the mundane, laborious and repetitive work, frees up the user to focus on more intellectually challenging tasks. This offers the potential to accelerate the discovery process by giving researchers more time to consider which compounds should be made. Therefore, adoption of our technology offers the potential to not only speed up each of the numerous iterative design-make-test cycles required in discovery research, but actually reduce the number of such cycles due to a greater amount of intellectual input.

\section{Expanding the Chemist's Toolkit with Automated Cartridge-based Synthesis}

There is a growing drive towards automation of chemical synthesis in industrial settings, with the majority of interest and endeavours primarily focused on the possibility of eliminating the human component of discovery research in all stages, using robotics, computational and machine learning/AI approaches. Such examples include efforts towards closed loop automation systems for drug discovery, ${ }^{[6]}$ in which the human factor is completely replaced and every single step of the whole discovery and process is automated and integrated.

The development of an effective closed-loop automation system for drug discovery is a grand goal, which offers so much promise. However, this is no easy task, which is evidenced by the fact that such systems are not yet being used routinely across the industry. The drive towards building a more capable automated system that can carry out many different manipulations and complicated processes sounds logical but such functionality results in extremely complex systems composed of many individual parts, all with the potential to fail. In addition, such complex systems need to be constantly monitored by an operator who needs to be highly skilled and able to identify and repair failed components and software issues.

The success of new technologies is dependent on whether a real need is being addressed and the rapid and wide adoption. While it is clear that there is a need for automation in chemical synthesis, adoption is often hindered because the typical, traditionally trained synthetic or medicinal chemist generally has neither the time or skills to dedicate to the development and operation of highly complex systems, since their primary focus is making new compounds for testing as quickly as possible, in order to progress the discovery project. Many chemists have experienced disappointment with automation and can recall their previous frustrations of working with a technology that actually makes their life more difficult, under the disguise of helping them out.

In an article published in 2019 by $\operatorname{Pan}^{[7]}$ the author mentions that, "the science of chemistry is not advanced by having robots do tasks that would otherwise be done manually." [...] Innovation [could even] potentially be decreased as chemists prioritize simple, automated workflows over more complex, manual procedures. Encouraging chemists to perform more routine chemistry is unlikely to provide any competitive advantage." This view stands to reason when considering large automation robots that need minimal human input once established but which require significant investment and effort to set up initially. One could then imagine that, once such technology has been established, there would be a bias towards processes suited to this technology. However, we suggest that alternative automation strategies do not necessarily lead to this same outcome.

One example of a value-adding tool for chemists, in which a routine, manual workflow has been automated, that has been very successfully integrated, is the 'automated flash column chromatography' range of machines. The first such devices were introduced around 25 years ago and were initially viewed with a great deal of caution and even scepticism by some users. Today however, they can be found in almost every synthetic chemistry laboratory and have replaced most of the manual column chromatography routinely done. These instruments, which still require a small amount of manual input, automatically carry out a single task with a high degree of reliability, have become very easy to use, thus offering real time-saving benefits. As such, their popularity has grown very rapidly in recent years.

It is, therefore, important that a range of complementary technologies are developed that offer chemists a broad automation toolkit from which they can select the most appropriate tool for the job. While the complex, closed-loop systems genuinely do have an important role to play in drug-discovery, these are perhaps better suited to the specialist technology groups supporting drug discovery efforts. However, for the majority of traditionally trained synthetic or medicinal chemists who continue to work with standard manual methods in the lab next door, a more appropriate tool might be one which is much less complex and easier to use. More importantly, those chemists can be confident that they can start the instrument and walk away without regretting it. Even systems like ours, which still require a small amount of manual input, can still provide valuable tools for synthetic and medicinal chemists since they automate critical and time-consuming steps.

Ultimately our aim is to provide a modular technology that will expand the chemist's available toolkit and serve as an ena- 
bling tool, allowing them to eliminate routine steps, free up more time for intellectual input or more time to spend on challenging reactions that are harder or even impossible to automate, and focus their efforts in a more effective and productive way. Using this approach, we can reap the benefits of humans and machines working together in partnership and provide research organisations with a competitive advantage. Although, there are many different approaches to automating chemistry processes, including enabling tools such as ours, there is unlikely to be a single approach that is suited to all situations, hence the need for a broad automation technologies toolkit that can be assessed and appraised by users for their individual needs.

\section{The Future of Automated Cartridge-based Synthesis}

The key to success of any new technology is that it solves an existing problem and is both easy to use and accessible. Our approach to promoting the adoption of this enabling technology has centred around flexibility and giving customers easy access to the synthesis technology. As such, customers can access the technology by opting for one of two different purchasing models. Either they obtain the instrument on a Pay Per Use basis, where the machine is effectively free of charge and the user pays a premium for the cartridges, or the machine can be purchased and customers can then profit from reduced prices on the cartridges ordered. In addition, Synple provides a service by which customers without a machine can place orders for compounds to be synthesised using specific cartridges and our in-house synthesiser farm.

In order to ensure the longer-term success of the technology, and keep solving existing problems, our aim is to continue developing new applications for reaction classes that are of value to the chemistry community, as well as those of interest to non-chemists in the discovery research field. In addition to our existing reaction portfolio, we have many more in the pipeline, including amide and azide forming reactions (Fig. 8).
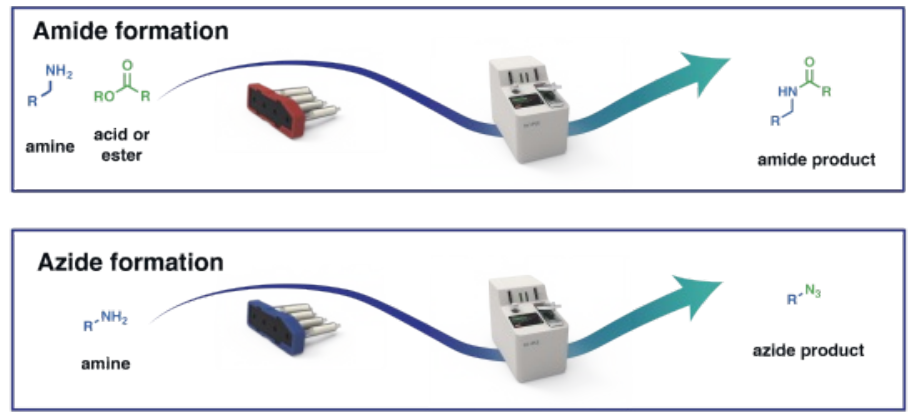

Fig. 8. Examples from our pipeline of new applications

Synple aims to address the growing need for easy to use, readily accessible automated synthesis solutions that have the potential to reduce synthetic bottle-necks and expedite the discovery of new materials and medical treatments in a more efficient and lower-cost manner. In addition, technologies, such as Synple's, that not only remove much of the manual work but that also enable easier access to new chemical space provide a real advantage to customers, who, through use of the technology, have the opportunity to create new IP, minimise the risk of costly research, and perhaps most importantly, develop new candidates for so far undruggable targets, enabling patients to benefit from crucial new treatments for current unmet medical needs. Such a step change in the way that organic synthesis is done could prove to be a major industry disruptor and give rise to the elusive productivity increases that are desperately needed.

\section{Synple Chem AG}

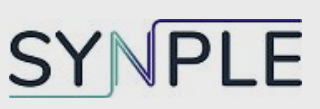

Synple Chem has developed an automated reagent cartridgebased synthesiser for the preparation of chemical compounds in chemical laboratories e.g. drug discovery laboratories, which works in an analogous manner to a very well-known coffee capsule system. With our device researchers can synthesize new molecules much faster, cheaper and more efficiently than using manual methods. This has the potential to speed up drug discovery cycles in the pharmaceutical industry.

\section{Key Figures}

Founding Year 2016

Location(s) Headquarter: Zurich Production: Zurich

Legal Form AG

Founders Dr. Benedikt M. Wanner Prof. Jeffrey W. Bode Dr. Paula L. Nichols Dr. Tuo Jiang Dr. Vijaya Pattabiraman

No of employees 5

Market capitalization

\section{Key Inventions}

Reagent Cartridges

Synple's reagent cartridges contain all reagents required for a particular chemical transformation, and include the reaction program stored on an RFID chip imbedded in the cartridge.

Synple 2

Synple 2 - The newest generation of our automated cartridge-based synthesiser is now available and works with all available reagent cartridges.

\section{Next Milestones}

October 2020

New reagent cartridges will be available, e.g. fluorinations, amide formations and more protecting group transformation chemistries.

June 2021

New generation of parallel synthesiser will be released.

Awards (or other important information)

December 2016 Venture Kick

Venture Kick's jury selected Synple Chem as one of the winners in first stage.

March 2018

\section{Science4Life}

Winner in the business plan competition of one of the oldest and most important science based startup competitions in Germany 
February 2019

InnoBooster - Gebert Rüf Stiftung

Funding support for Synple Chem, boosting the development of further applications.

\section{Contact}

Contact Person

Benedikt Wanner, CEO

\begin{tabular}{l|l} 
Address & $\begin{array}{l}\text { Synple Chem AG } \\
\text { Vladimir-Prelog-Weg 3 } \\
8093 \text { Zurich }\end{array}$ \\
\hline E-mail & info@ synplechem.com \\
\hline Website & www.synplechem.com
\end{tabular}

\section{Acknowledgements}

We would like to thank the whole Synple Chem team and investors who made this success possible. Also we are very grateful for the support given by everyone involved at ETH; the ETH Transfer team, the LOC machine shop (Christoph Bärtschi) and all our students who contributed to the technology (Oliver Groninger, Leran Zhang, Konrad Leopold and Ralph Werner)

Received: June 30, 2020
[1] a) E. De Clercq, Med. Res. Rev. 2015, 35, 698; b) J. Mao, H. Yuan, Y. Wang, B. Wan, M. Pieroni, Q. Huang, R. B. van Breemen, A. Kozikowski, S. G. Franzblau, J. Med. Chem. 2009, 52, 6966.

[2] a) T. Tsukamoto, ACS Med. Chem. Lett. 2013, 4, 369; b) V. A. Steadman, ACS Med. Chem. Lett. 2018, 9, 581.

[3] a) C.-V. T. Vo, G. Mikutis, J. W. Bode, Angew. Chem. Int. Ed. 2013, 52, 1705; b) M. U. Luescher, K. Geoghegan, P. L. Nichols, J. W. Bode, Aldrichim. Acta $\mathbf{2 0 1 5}, 48,43$.

[4] M. Xi, Y. Chen, H. Yang, H. Xu, K. Dui, C. Wu, Y. Xu, L. Deng, X. Luo, L. Yu, Y. Wu, X. Gao, T. Cai, B. Chen, R. Shen, H. Sun, Eur. J. Med. Chem. 2019, 174, 159 .

[5] For a full set of detailed application notes see: http://synplechem.com/solutions

[6] a) D. M. Parry, ACS Med. Chem. Lett. 2019, 10, 848; b) S. M. Pant, A. Mukonoweshuro, B. Desai, M. K. Ramjee, C. N. Selway, G. J. Tarver, A. G. Wright, K. Birchall, T. M. Chapman, T. A. Tervonen, J. Klefström, J. Med. Chem. 2018, 61, 4335.

[7] J. Pan, ACS Med. Chem. Lett. 2019, 10, 703.

\section{License and Terms}

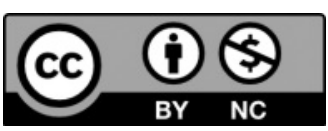

This is an Open Access article under the terms of the Creative Commons Attribution License CC BY_NC 4.0. The material may not be used for commercial purposes.

The license is subject to the CHIMIA terms and conditions: (http:// chimia.ch/component/sppagebuilder/?view=page \&id=12).

The definitive version of this article is the electronic one that can be found at https://doi.org/10.2533/chimia.2020.808 